\title{
Numerical Simulation of Supersonic Flow through Scramjet Intake with Concavity in Cowl Surface
}

\author{
P. Senthilkumar ${ }^{1,2_{\dagger}}$ and T. M. Muruganandam ${ }^{1}$ \\ ${ }^{I}$ Department of Aerospace Engineering, Indian Institute of Technology, Madras - 600036, India \\ 2. School of Mechanical Engineering, Vellore Institute of Technology, Vellore, India
}

$\dagger$ Corresponding Author Email: senthilp76@gmail.com

(Received October 1, 2019; accepted March 19, 2020)

\begin{abstract}
Scramjet intake usually employs shock waves to reduce the flow velocity and increases the static pressure of the flow. However, this causes flow separation and multiple reflections of shock waves, which result in total pressure loss for the flow. This paper discusses the performance enhancement of scramjet intake through the implementation of a concavity along the cowl surface. The baseline intake model used here is the same as that reported in Emami et al. (1995) Two models with the concavities of depth 0.05 and 0.1 inches on cowl inner surface are numerically simulated at Mach number 4.03, and compared with the base model. An improvement in the performance is investigated in terms of total pressure and flow separation. Present study shows that a concavity on cowl surface reduces the flow separation on the ramp wall and increases the total pressure when compared to the base case. This is achieved by expansion fans produced at the beginning of the concavity. These expansion fans weaken the cowl lip shock and suppress the separation size. Further, it turns the shock waves along the flow, decreasing the number of shock wave reflections in the isolator. Thus, increase in total pressure at the exit of the isolator is observed. It is found that there is a marginal increase in Mach number for both the concavity cases without any change in mass flow rate. There was a minor flow distortion observed, which may be corrected by changing the isolator length. This study demonstrates the scope of overall improvement in scramjet engine performance by implementing concavity along the cowl surface.
\end{abstract}

Keywords: Scramjet intake; Concavity; Flow distortion; Expansion fans; Shock wave boundary layer interaction; Total pressure recovery.

\section{NOMENCLATURE}

$B \quad$ baseline model

$C_{l} \quad$ model with concavity of 0.05 inch depth

$\mathrm{C}_{2}$ model with concavity of 0.1 inch depth

$\mathrm{H}_{\mathrm{t}}$ throat height

\section{INTRODUCTION}

Scramjet engines are not having any rotating components like compressors and turbines. The combustor is designed to have combustion in supersonic flow with sufficient static pressure, maximum possible total pressure and minimum flow distortion. Intake is designed in such a way that the required static pressure rise is obtained by compressing the incoming free stream of air by series of oblique shocks. The pressure rise is obtained from each shock wave and same time there is considerable total pressure loss also.

An undesirable phenomenon called shock wave boundary layer interaction (SWBLI) which takes $p \quad$ static pressure

$p_{\infty} \quad$ free stream static pressure

$x \quad$ distance along $\mathrm{x}$ direction place on the ramp/cowl surfaces. When the shock waves are impinging on the surfaces which already have boundary layer growth, creates an adverse pressure gradient for the boundary layer. This forms thickening of boundary layer and followed by separation. This SWBLI leads to unwanted effects like decrease in mass capture, total pressure loss, increased drag, etc. This may even go to the extent of unstarting of the engine too.

Emami et al. (1995) parametrically tested inletisolator model of an airframe-integrated engine of ramjet/scramjet in the coldflow Mach 4 Blowdown Facility (M4BDF) at Langley Research Centre. Model undertaken for his extensive study involved different lengths of rotating inlet cowls and 


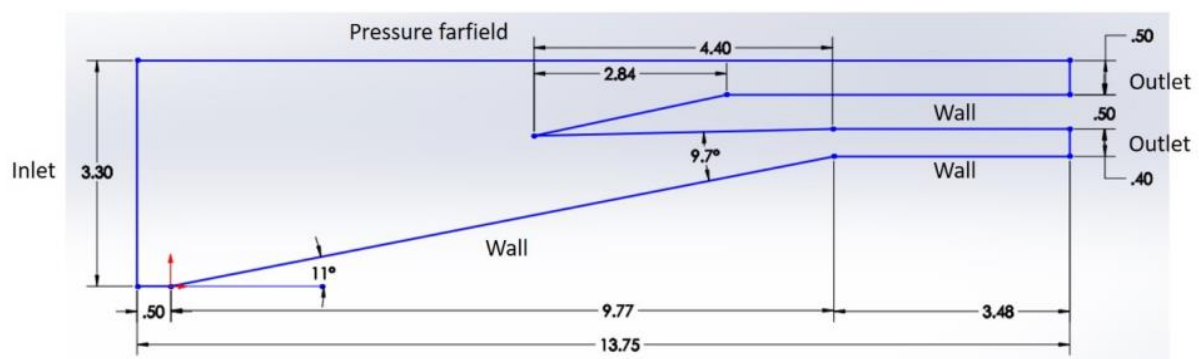

Fig. 1. Intake Geometry Base line (B) (adopted from Emami et al. 1995).

interchangeable isolator sections. Several combinations of cowls and isolator sections lead to the formulation of 250 more geometric arrangements. He provided start and unstart phenomenon with possible pressure data. Later Janarthanam and Babu (2012) numerically validated the combinations obtained with three different cowl lengths and five different cowl angles in Mach 4.03 flow. In addition to pressure distribution data, he presented flow field images like shock structure, shock wave boundary layer interaction, start and unstart situations. Surujhlal and Skews (2018) investigated both experimentally and numerically the supersonic flow over concave planar, symmetric surfaces and verified curved shock theory. He studied the effect of geometric curvature on the shock structure downstream in a supersonic flow. Filippi and Skews (2017) studied the effects of internal surface curvature and leading-edge angle on the shock waves. He noticed that steeper compression fans were formed when both the internal curvature and leading edge angle high. These fans had an effect on the trailing edge expansion fans and thus the downstream shock structure has been modified. Wan and Guo (2007) and Waltrup et al. (1982) tested a fixed-geometry two-dimensional mixed-compression supersonic inlet with sweep-forward high-light and bleed slot in an inverted "X"-form layout and presented the changes in total pressure recovery, mass flow rate with different angles of attack. Raja Sekar et al. (2019) in his numerical study with perforated wall in the entire isolator concluded that considerable reduction in separation bubble is possible. Creighton and Hillier (2007) studied hypersonic flow through annular cavities with Mach number 8.9 both experimentally and numerically. $\mathrm{He}$ suggested a parameter to differentiate between weak and strong oscillations with a help of several cavities of varying length-to-depth ratios. Lawson and Barakos (2011) reported in his review of turbulent cavity flows that the importance of unsteady pressure measurement and time resolved flow field images data. This could help to understand complex flow filed happening in cavities particularly in high speed air intakes. Palharini et al. (2018) in his numerical simulation studied the effect of cavities particularly 3D in chemical reaction environments as well as rarefied environment. He explored his study with different length to depth ratio cavities and reported the stagnant situation of particle inside the cavity and recirculation regions. Mccormick (1993) compared the performance of micro vortex generator and passive cavity in controlling the flow separation. He proved that passive cavity is suitable to reduce drag most and vortex generators are suitable to reduce shock induced separation in supersonic flows. Morgenstern and Chokani (1994) observed the oscillatory behavior of supersonic flow over cavity and its behavior with Reynolds number with a help of unsteady pressure data and power spectral analysis. Mohri and Hillier (2011) Guvernyuk_et al. (2016) and Heiser et al. (1994) reported the supersonic flow behavior over axisymmetric cavities and flow transition from open to closed type for different length to depth ratio. Reports are available for supersonic flow filed over serrated edge cavity (Gai et al. 2015) and sub cavity (Lad et al. 2018, Panigrahi et al. 2019). In order to have better flow control, micro jet injection in the cavity was experimentally done by Zhuang et al. (2006).

Thus many studies are available to understand the effects of geometry modifications in scramjet intakes including cavities. In this research work, cowl is altered to form curved surfaces of different depths at a fixed distance from the leading edge. This is expected to decrease the strength of the cowl shock with some expansions, and replace it with a set of weak compression waves later. The ability of the modification to decrease separation losses, and along with it, the pressure recovery, and therefore, its effects on the overall efficiency of scramjet is studied.

\section{MODEL DETAILS COMPUTATIONAL METHODOLOGY}

Intake baseline geometry considered for this study is the same as that experimentally tested by Emami et al. (1995) and numerically simulated by Janarthanam and Babu (2012). The intake model consists of compression ramp, isolator and cowl. Sketch for intake model with dimensions is given in Fig.1. The compression ramp is at an angle of $11^{\circ}$ with respect to horizontal along with a length of 9.77in. End of inclined ramp is connected to a flat surface known as isolator having a length and height of 3.48in and 0.4in respectively. Total computational domain is $13.75 \mathrm{in}$ long in $\mathrm{x}$-axis and 3.3in high in y-axis. The cowl is connected to a hinge at a height of 0.4in above the end of 
compression ramp and is allowed to rotate at different angles. In the present study, cowl having a convergence angle of $9.7^{\circ}$ to the ramp and a length of 4.4 inch is adapted.

Turbulent Reynolds-average Navier-Stokes (RANS) equations are solved in the present numerical simulations. In order to have the closeness in the calculations of continuity, momentum and energy equations, the working fluid is taken as ideal gas. Calculation of viscosity is carried out using Sutherland three coefficient method. Mach number is taken as 4.03 whereas free stream static and stagnation pressure of $8724 \mathrm{~Pa}$ and $1.31 \mathrm{MPa}$ respectively. No slip and adiabatic conditions are applied at all walls. All inlets are considered as a supersonic inlet by making them pressure far field whereas outlets are pressure outlet. Free stream temperature is taken as $69.15 \mathrm{~K}$ while $288 \mathrm{~K}$ is the stagnation temperature. Two equation SST K- $₫$ is adapted for turbulence modelling. Advection Upstream Splitting Method (AUSM) explicit scheme has been used as a solver. It involves a numerical inviscid flux function for solving the conservation equations. In order to get more accuracy in high speed flow simulations, the flow type is set to be second order upwind. Turbulence intensity is kept at 5\% initially. It is then varied between $5 \%$ and $15 \%$ and simulations were carried out individually. It is found from the static pressure distribution along the ramp and cowl, change in turbulence intensity did not change the shock structure much. It is also compared with skin friction coefficient plots. The variations are very negligible. Hence turbulent intensity $5 \%$ is maintained for all the simulations in this research work.

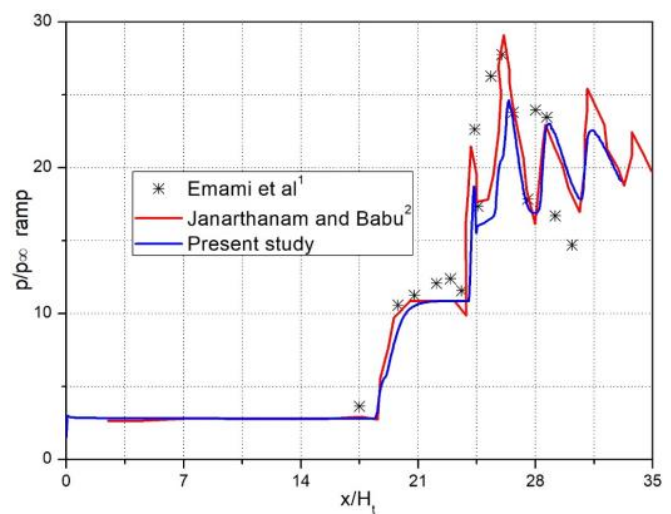

Fig. 2. Static pressure distribution along ramp.

Numerical simulations are carried out for 2D geometry for this intake model and static pressure distribution along the ramp is plotted in Fig. 2. This plot is compared with static pressure plots obtained from both Emami et al. (1995) and Janarthanam and Babu (2012) It is observed that, pressure plots are nearly matching. The error is less than $10 \%$, and is capturing all the major features of the flow. Also, contours of density gradient from computations is plotted in Fig. 3 and are compared to that of Janarthanam and Babu (2012) in Fig. 4. The experimental flow field images are not available and the start and unstart situations were predicted by pressure data only (Emami et al. 1995). Hence, in the present study the numerical schlieren image is compared with the numerical schlieren image presented by Janarthanam and Babu (2012). It is evident that the separation bubble and shock structure obtained in the present study agrees well with that from Janarthanam and Babu (2012) as shown in Figs. 3 and 4.

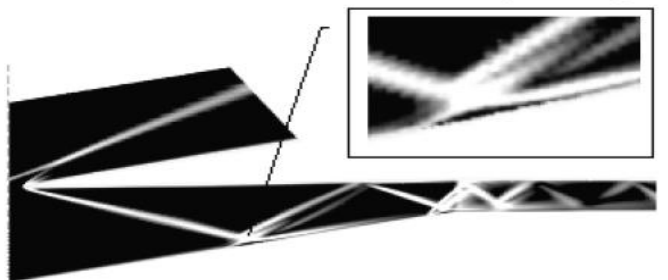

Fig. 3. Contours for density gradient with SWBLI zoomed in (Janarthanam and Babu 2012).

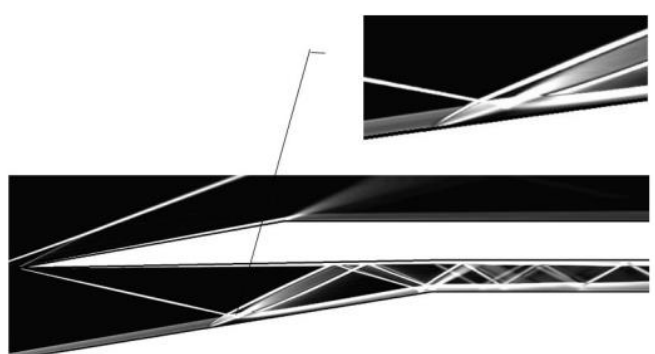

Fig. 4. Contours of density gradient with SWBLI zoomed in (present study).

\subsection{Grid Independence Study}

Initially, mesh with 347900 elements is created keeping fine mesh near the ramp surface. The results obtained from this mesh shows a y+ value of 19.97 at the ramp. To capture the boundary layer, mesh should be as fine as possible near the ramp. Accordingly, geometry with 727950 elements is simulated and an average $y+$ value of 8.66 is reported. To get more accuracy, mesh is further refined to 1240500 elements. This case is assumed to be the most appropriate when compared with other two cases as the y+ value decreased to 4.5. Thus, mesh having 1240500 elements is adapted for all further simulations. Also, static pressure distribution along the ramp as well as along the cowl is plotted in Fig. 5 and Fig. 6 respectively. For all cases it is observed that the deviation in pressure values is less than $1 \%$. Hence, it clearly signifies that results are grid independent.

Numerical schlieren images presented in Fig. 4 clearly shows an oblique shock originating from the leading edge of the ramp and a small gap between the shock and cowl lip is seen. This supports sub critical mode of operation. This mode of operation will not provide maximum mass flow rate whereas critical mode of operation supports maximum mass flow rate. But this is a unique situation and difficult to maintain too. 
A shock wave boundary layer interaction (SWBLI) is found along the ramp surface. This is due the impingement of the shock from the cowl lip on the ramp surface where boundary layer developed already. The separation shock and reattachment shock are clearly seen on the ramp surface. These shocks are getting reflected from the cowl bottom surface and directed towards ramp. Even though these shocks are impinging on the boundary layer on the ramp surface, SWBLI is not found. The shock strength and shock angle are not favorable to create shock wave boundary layer separation (Needham and Stollery 1965, 1966). These two shocks are reflected back as single shock wave to the upper portion of the isolator as seen in the Fig. 4. There is a weak shock wave emanating from the junction of the cowl - isolator. This is weakened on further reflections inside the isolator. Further shock reflections are seen in the isolator. The isolator length is designed in such way that not to have normal shock anywhere inside the isolator. Supersonic flow is available at the exit of the isolator which will support for combustion. Thus the shock system in the compression region and in the isolator section are found to be the same between the validation case (Janarthanam and Babu 2012) and the present study. The pressure peaks inside the isolator due to the shock reflections are clearly seen from the pressure distribution plots (Fig.5 and Fig.6) and closely agreeing with the experimental data (Emami et al. 1995) and numerical simulation data (Janarthanam and Babu 2012)

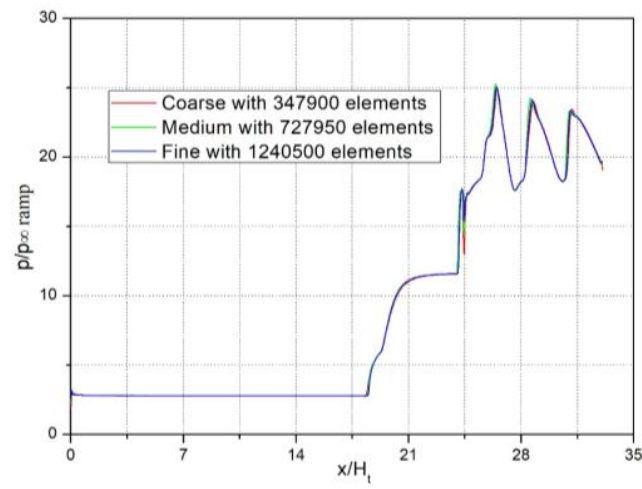

Fig. 5. Static pressure distribution along ramp surface for all three types of mesh.

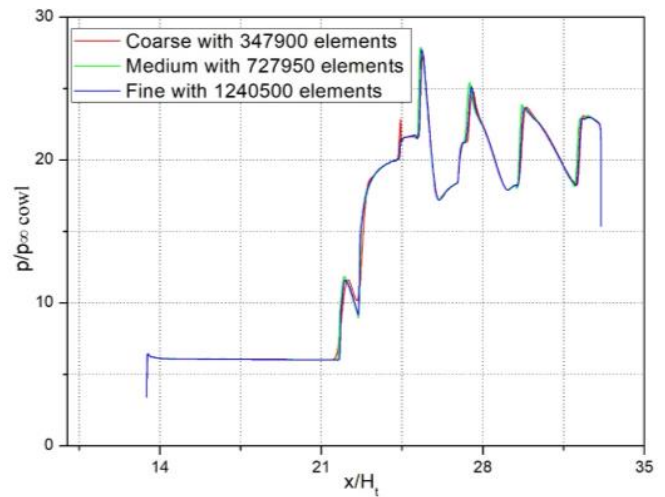

Fig. 6. Static pressure distribution along cowl for all three types of mesh.

\section{PERFORMANCE IMPROVEMENT BY GEOMETRIC MODIFICATIONS}

The design of inlet and isolator is carried out to give possible static pressure rise as well as minimum total pressure loss. This is achieved by compression produced by series of oblique shock waves both in inlet and isolator. Since combustor is designed to have combustion at supersonic speed, airflow at isolator exit should be supersonic. At the same time care should be taken to have minimum total pressure loss and flow distortion. These parameters decide the performance of any intake system. The performance improvement is done by several methods. Geometric modifications is used to improve the performance of the inlet isolator in this research work. As discussed earlier, the static pressure rise is achieved by series of oblique shocks in inlet and isolator. For considerable compression ratio, the shock required will be strong and this can cause boundary layer separation and total pressure loss. This is severe when the shock is strong at higher Mach number, this study relaxes this problem by decreasing the shock strength when it interacts with the wall, but will introduce a set of weak compression waves along the concave surface. This is the idea of introducing the concavity in the cowl inner wall. The concavity starts slightly downstream of the cowl tip so as not to affect initial part the cowl lip shock. It is expected that the expansion created by the concavity will decrease the strength of the cowl shock before it reached the ramp and thus alleviate its effect on the boundary layer. The change in performance due to geometric modifications in terms of the changes in wall static pressure, total pressure recovery, flow separation and Mach number are studied.

Cowl inner surface of the adopted model is created with concavity of various depths. The concavity is basically a circular arc cavity starting from 0.5 inch downstream from the leading edge of the cowl and ending at the throat, with different depths. In this paper, two cases are adopted with 0.05 inch and 0.1 inch depth as shown in the Figs. $7 a$ and $7 b$ respectively. The performance is compared with zero depth cavity (baseline) case.

All other dimensions remain the same as base model. Numerical simulations are individually performed with the above modifications in the geometry. The numerical schlieren obtained for each case is presented in Fig. 8. All the images are arranged in a common frame to compare the shock angles, the changes in the shock impingement locations on both ramp and cowl surfaces. These numerical schlieren are basically density gradients. Shock impinging locations are marked as 1,2, 3, up to 11 in the base model. Similarly the same are marked as 1', 2' 3' up to 11' for concavity model of depth 0.05 inch and 1", 2", 3" up to 9" for concavity model of depth 0.1 inch.

The base model shock train is clearly seen in Fig. $8 \mathrm{a}$. The presence of the concavity changed the 


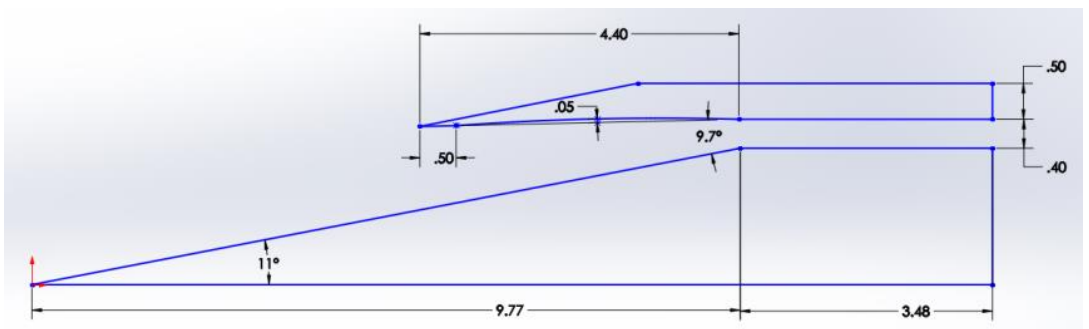

Fig. 7a. Intake geometry with concavity of depth 0.05 inch (C1).

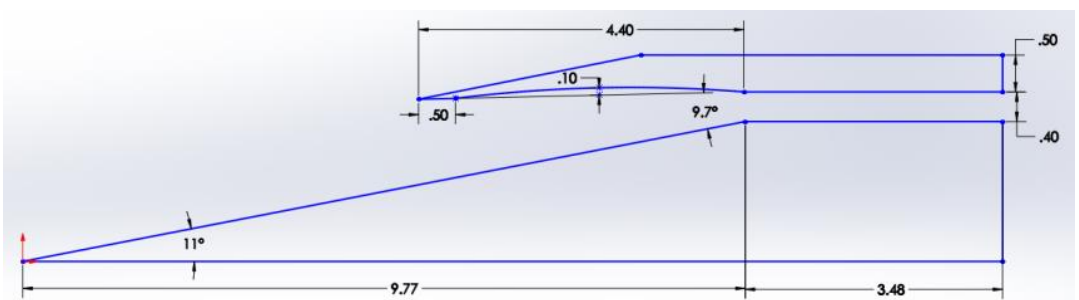

Fig. 7b. Intake geometry with concavity of depth 0.1 inch (C2).
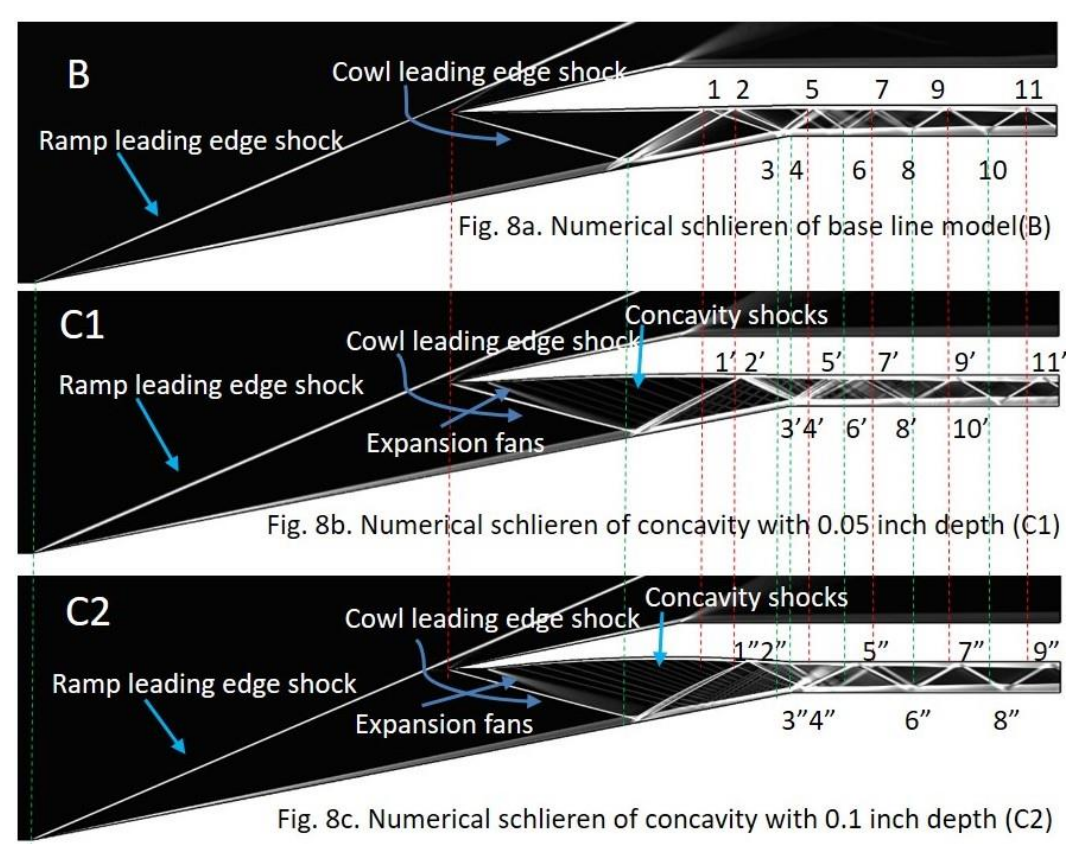

Fig. 8. Numerical schlieren of cases $\mathrm{B}, \mathrm{C} 1$ and $\mathrm{C} 2$.

shock structure as seen in the other modified geometries as shown in Figs. 8b and 8c. The cowl lip shock impinging location on ramp surface of both the concavity models are not same when comparing with the base model. The separation shock in the base model emanates from the ramp surface and impinges on the cowl inner surface at location 1 as shown in Fig. 8a. The reattachment shock emanates from the ramp surface and impinges on the cowl inner surface at 2. Both shocks reflect back to the ramp surface. Thus the compression region has five shocks design. This can be seen clearly in Fig. 8a. The subsequent shock reflections are seen in the isolator portion and they are numbered from 3 to 11 as seen in Fig. 8a. The development of boundary layer and its gradual growth in the inlet compression portion and in the isolator are also captured in the numerical schlieren.

The impingement location of the cowl lip shock on the ramp surface in cases $\mathrm{C} 1 \& \mathrm{C} 2$ are not same as case B. Expansion fans are evident at the beginning of the concavity in cases $\mathrm{C} 1 \& \mathrm{C} 2$. When these expansion fans interact with the cowl leading edge shock, it is curved towards the flow direction. Hence the impinging location on the ramp surface is moved along the flow direction as shown in Figs. $8 \mathrm{~b}$ and $8 \mathrm{c}$. This curving of the shock towards the flow direction decreases the shock strength. Considerable reduction in the separation bubble size is also noticed in the modified cases. Among the concave cowl cases, case $\mathrm{C} 2$ has smaller separation bubble compared to that of case $\mathrm{C} 1$. While the 
figure is not very clear, it is evident from the reattachment shock getting closer to the separation shock. The separation shock angle and reattachment shock angles are reduced and their impingement locations on the cowl surface $\left(1^{\prime}, 2^{\prime}\right.$ and $\left.1^{\prime \prime}, 2^{\prime \prime}\right)$ shifted more downstream. The shock impingement locations in the isolator section are shifted towards the downstream side as shown in the Figs. $8 \mathrm{~b}$ and $8 \mathrm{c}$. This is actually the result of weaker cowl lip shock whose reflections form the shock train. Overall reduction in static pressure rise is expected due to this at the isolator exit, compared to case B. Nevertheless, the reduction in the separation is expected to give a more positive impact (in terms of total pressure and Mach number) which will be shown later.

\section{SHOCK WAVE BOUNDARY LAYER INTERACTION}

Figure 9 shows the enlarged view of numerical schlieren of the inlet shock interactions for the three cases. The shock wave boundary layer interaction in the base model is clearly captured. The separation shock, expansion fans and reattachment shock from the separation bubble are clearly seen in the Fig. 9a.

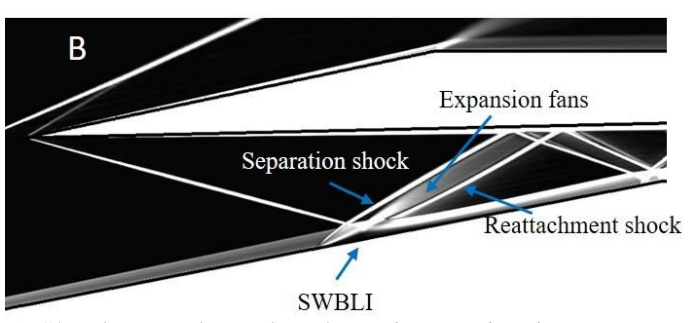

a) Shock wave boundary layer interaction in case B

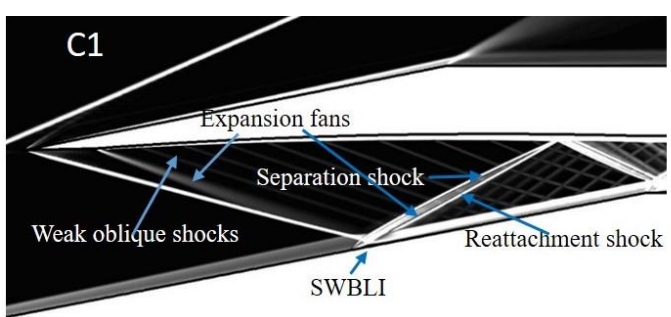

b) Shock wave boundary layer in case C1 (present study)

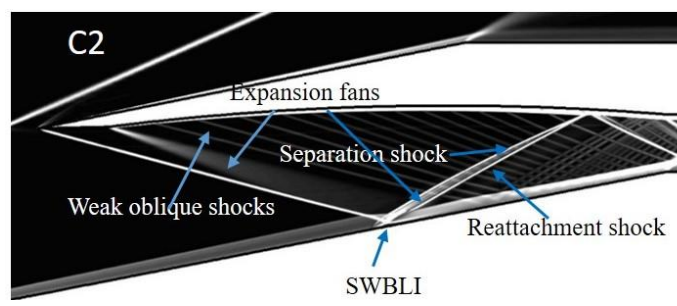

c) Shock wave boundary layer interaction in case C2 (present study)

Fig. 9. Shock wave boundary layer interaction.

It can be seen from the schlieren images that the model $\mathrm{C} 2$ has the smallest separation bubble. This is due to the expansion fans produced at the beginning of the concavity. These expansion fans weaken the cowl lip shock and decreases the adverse pressure gradient. This also decreases the gap between the separation and the reattachment shocks, causing them to hit almost the same location on the top wall. It is also observed that the compression waves from the concavity surface are inclined towards downstream direction. These two effects together cause the shock train to become more inclined towards the downstream direction. These effects are more pronounced in the $\mathrm{C} 2$ case than $\mathrm{C} 1$ case. The $\mathrm{C} 2$ case has lesser number of oblique shocks downstream as the separation and reattachment shocks come closer. However, there are stronger weak compression waves in $\mathrm{C} 2$ than C1 case from the concavity. This is gives lower stagnation pressure losses as will be presented later. Another side effect of this geometric modification is that the flow is accelerated due to these modifications, and will be discussed later.

Shear stress along the flow direction on the wall are plotted for the three cases in Fig. 10. The negative values for the wall shear stress is seen where the flow is reversed. It can be seen first that the point of separation is moved more downstream for the two modified cases compared to the baseline case. The length of the negative shear stress region is lower for $\mathrm{C} 2$ than $\mathrm{C} 1$ by a marginal amount. Thus one can say that the losses due to separation are decreased significantly due to the concavity. One can also notice the effect of individual weak compression waves from the concavity on the wall shear stress just downstream of the separated region.

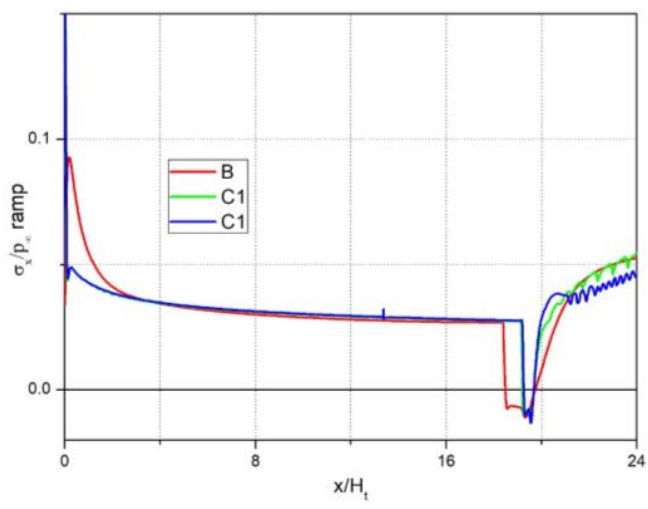

Fig. 10. Wall shear stress along the ramp surface.

\section{STATIC \\ PRESSURE DISTRIBUTION ALONG RAMP AND COWL SURFACES}

The static pressure distribution along the ramp surface and cowl surface are shown in the Fig. 11 and 12 respectively for cases $\mathrm{C} 1$ and $\mathrm{C} 2$, respectively, along with those for the baseline case. The numerical schlieren image of the modified case is positioned in between the plots of static pressure distribution along the cowl and the ramp. The pressure peaks corresponding to the shock impinging locations are shown by extending a line 
from the point of impingement. It is evident that the pressure drops near the start of the concavity, and then increases gradually due to the weak compression waves in the concave region. The expansion from the end of the concavity and the reflected shock from the cowl both interact near the throat and negate each other causing only a small kink in the pressure on the ramp wall. All the pressure peak values are lower than the pressure peak values of base case. The downstream shift in the impingement locations of the shock train are also seen clearly in all the pressure plots. The base model shocks have a sharp impinging location on the cowl surface whereas the modified cases do not have sharp impinging locations. It can be seen in the plots that pressure peaks are sharp in base model and slightly flatter in modified cases. This is due to the combined effects of the weak compression fans, the expansion fan from the throat, and the shock train from the cowl lip.
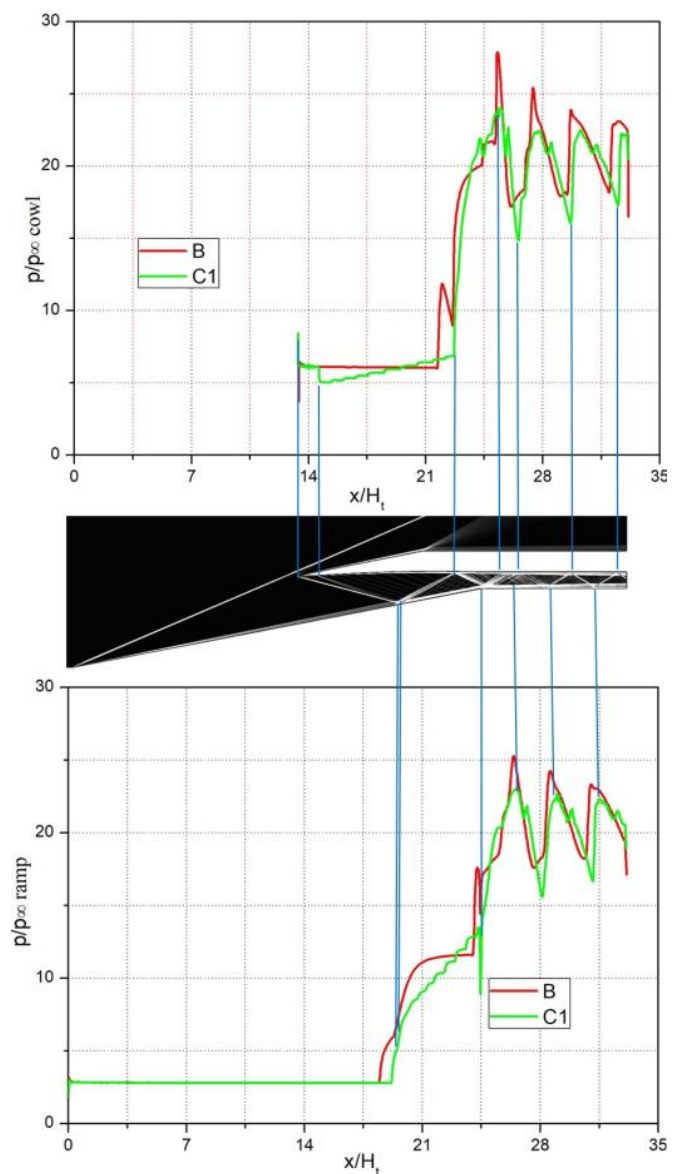

Fig. 11. Comparison of static pressure distribution along the ramp and cowl surfaces in cases $B$ and $\mathrm{C} 1$.

\section{STATIC PRESSURE VARIATION AT ISOLATOR EXIT}

The overall purpose of the scramjet intake is to deliver air to the combustor at required static pressure and Mach number. The combustor will perform better if there are no large spatial nonuniformities in the pressure and Mach number distributions at the end of the isolator. This must truly be achieved by ensuring that the shock train becomes weak and does not cause major fluctuations spatially. The static pressure variations across the isolator exit for the three cases are shown in Fig. 13. The pressure variations are according to the shock position at the exit. The static pressure rise for the modified cases are lesser than the base model. Since the number of shock waves are reduced and shock angles become weaker, (in order to decrease the total pressure loss) the static pressure rise is limited. It is noticed that rise in static pressure in upper region (higher y, or cowl side) is more than lower region. This is because of the shock in the shock train has just reflected off the top wall in all the cases. Among the modified cases, case $\mathrm{C} 1$ has better mean static pressure rise than the case $\mathrm{C} 2$. The mass weighted mean static pressure values are presented in table 1 .
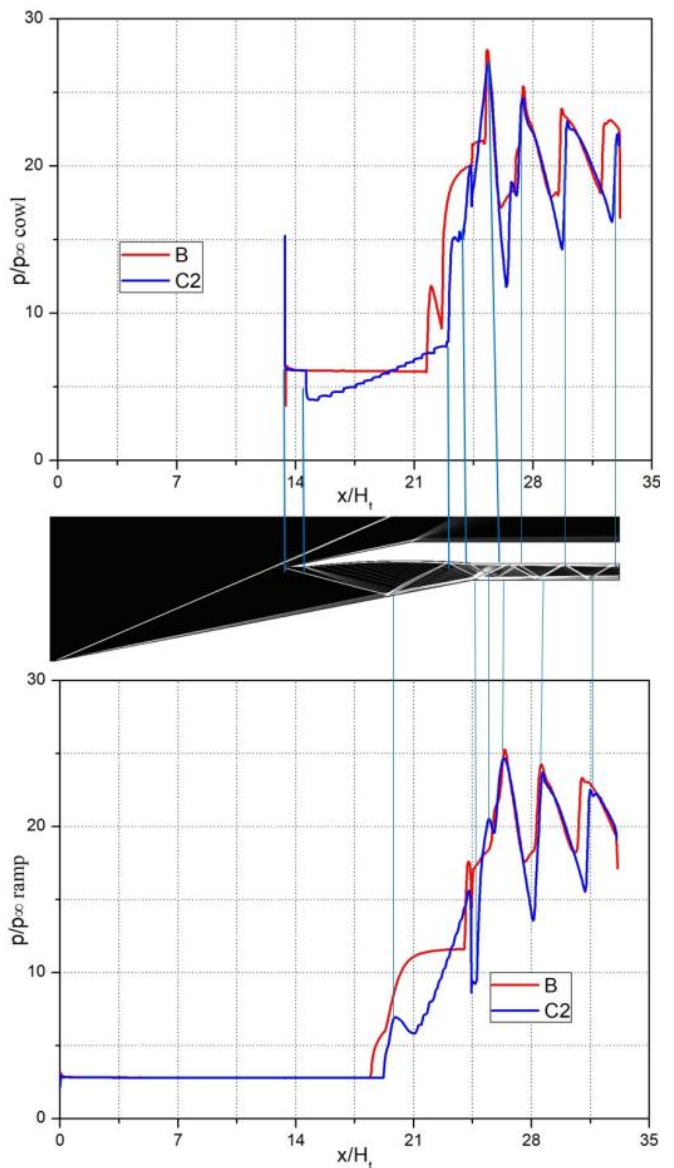

Fig. 12. Comparison of static pressure distribution along the ramp and cowl surfaces in cases $B$ and $C 2$.

Table 1 Mass weighted mean properties at the exit of the isolator

\begin{tabular}{|c|c|c|c|}
\hline Cases & B & C1 & C2 \\
\hline $\begin{array}{c}\text { Static pressure } \\
{[\mathrm{Pa}]}\end{array}$ & 184432 & 178657.32 & 172389.8 \\
\hline
\end{tabular}




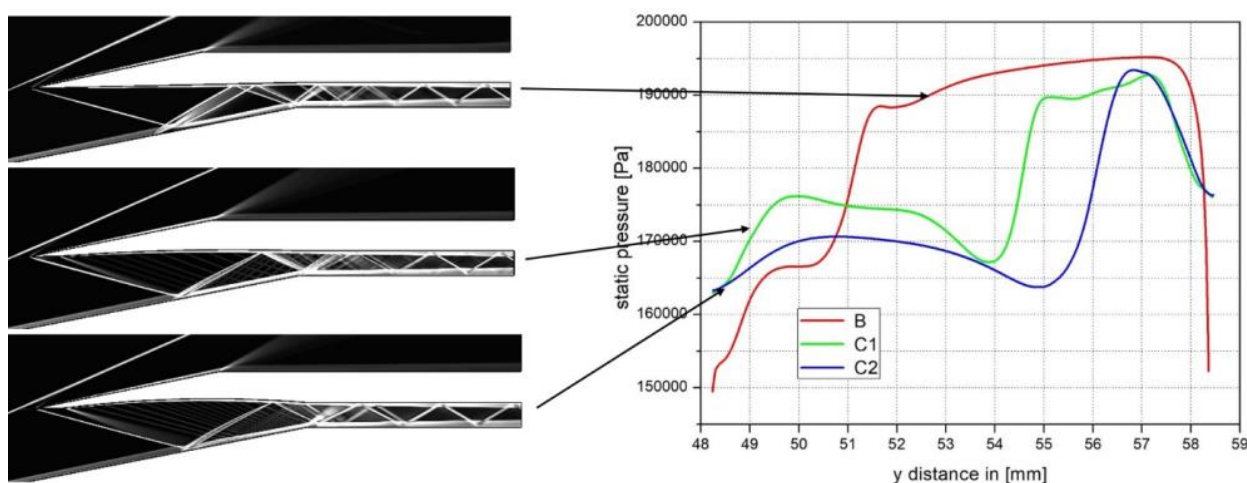

Fig. 13. Static pressure variation at isolator exit for all cases.

Table 2 Comparison of total pressure recovery and flow distortion

\begin{tabular}{|c|c|c|c|c|}
\hline \multirow{2}{*}{ Cases } & \multirow{2}{*}{$\begin{array}{c}\text { Total pressure } \\
\text { recovery }\end{array}$} & \multirow{2}{*}{$\begin{array}{l}\text { \% increase in } \\
\text { total pressure }\end{array}$} & & \multicolumn{2}{|c|}{ Flow distortion } \\
\cline { 4 - 5 } & & & $\begin{array}{c}\text { Based on } \\
\sigma^{2}\end{array}$ & $\begin{array}{c}\text { Based on } \\
p 0 \text { max }-p 0 \min \end{array}$ \\
\hline $\mathrm{B}$ & 0.71 & - & 0.56 & 0.93 \\
\hline $\mathrm{C} 1$ & 0.74 & 4.23 & 0.98 & 1.04 \\
\hline $\mathrm{C} 2$ & 0.75 & 5.63 & 0.87 & 1.01 \\
\hline
\end{tabular}

\begin{tabular}{|c|c|c|c|c|}
\hline \multirow{3}{*}{ Cases } & \multicolumn{2}{|l|}{ Flow distortion } & \multicolumn{2}{|c|}{ Flow distortion } \\
\hline & $\underset{\sigma^{2}}{\text { Based on }}$ & $\underset{\text { pomax - p0min }}{\text { Based on }}$ & $\begin{array}{l}\text { Based on } \\
\sigma^{2}\end{array}$ & $\begin{array}{c}\text { Based on } \\
p 0 \max -p 0 \min \end{array}$ \\
\hline & 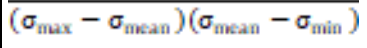 & pomean & $\overline{\left(\sigma_{\max }-\sigma_{\text {mean }}\right)\left(\sigma_{\text {mean }}-\sigma_{\min }\right)}$ & p0mean \\
\hline B & 0.56 & 0.93 & 0.56 & 0.93 \\
\hline C1 & 0.98 & 1.04 & 0.98 & 1.04 \\
\hline $\mathrm{C} 2$ & 0.87 & 1.01 & 0.87 & 1.01 \\
\hline
\end{tabular}

\section{MACH NUMBER VARIATIONS AT EXIT}

Another side effect of this geometric modification is that the flow is accelerated due to these modifications, and this is the reason for the static pressure drop at the exit plane. The scramjet combustor is designed to have supersonic flow for combustion. Change in Mach number can have adverse effects on the flame stability and thus this should be reported here. At the end of the intake, it is desirable to have uniform distribution of Mach number. Figure 14 compares the Mach number variations across the isolator exit for the three cases. Marginal increase in the Mach number is obtained in both the modified cases compared to the base case. Modified geometries have slightly higher Mach number over a considerable exit height. This will give more uniform Mach number profile for the combustor. Case $\mathrm{C} 1$ has a shock cutting the middle of the exit plane, causing the change in the middle. This may be avoided if the isolator length was modified. However, one should note that the shock train will continue into the combustor in all the cases. Thus the modified cases are better for the combustor since the shocks are a little weaker.

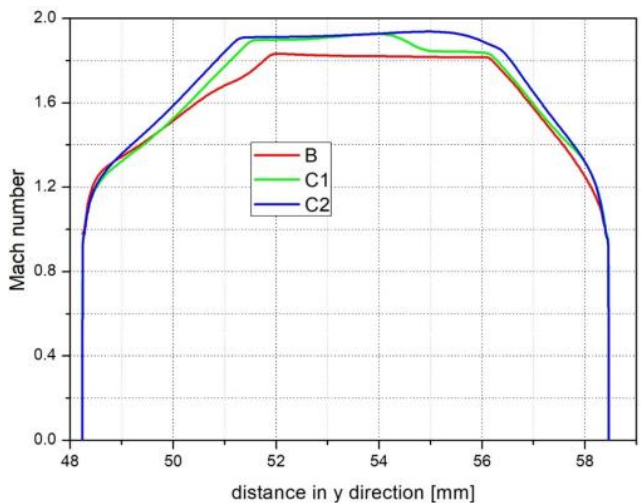

Fig. 14. Mach number variation at isolator exit for all cases.

\section{TOTAL PRESSURE RECOVERY AND FLOW DISTORTION}

In order to compare the overall performance of the intake-isolator geometry, the performance parameters stagnation pressure recovery and flow distortion 20 are calculated for both the cases. The design of any intake system is said to be good if the pressure recovery is high and flow distortion is low. The stagnation pressure recovery and flow distortion are calculated as follows. 
Stagnation pressure recovery $=\frac{p_{0 \text { avg }}}{p_{0=0}}$

Based on Liscinsky (1993)

Flow distortion $=\frac{\sigma^{2}}{\left(\sigma_{\max }-\sigma_{\text {mean }}\right)\left(\sigma_{\text {mean }}-\sigma_{\min }\right)}$

Based on Janarthanam and Babu (2012)

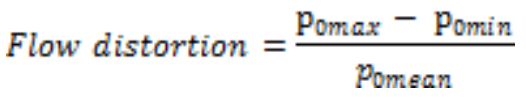

Where,

$\sigma$ - standard deviation of stagnation pressure,

$\sigma_{\max }-$ maximum stagnation pressure,

$\sigma_{\text {mean- }}$ mean stagnation pressure and

$\sigma_{\min }-$ minimum stagnation pressure.

These values are calculated for the base line case and the present study cases and given in the table. 2 .

The base case without any geometrical modifications gives possible total pressure recovery 0.71 . Among the modified geometries, $\mathrm{C} 1$ geometry has the rise in total pressure of $4.23 \%$ whereas $\mathrm{C} 2$ geometry has a total pressure rise of $5.63 \%$. Flow distortion is controlled by the exit plane shock location in the isolator. As discussed earlier, the flow distortion can be adjusted by the isolator length. However, one should keep in mind that the shock train continues into the combustor anyways.

The reduction in the shock strengths and separation bubble size helped to have total pressure rise in the modified cases. More total pressure recovery in case of $\mathrm{C} 2$ is due to the possible reduction in the separation bubble (Fig. 9).

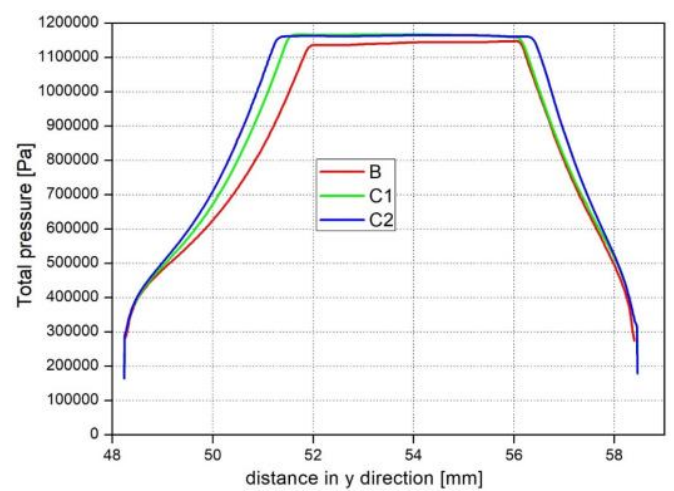

Fig. 15. Total pressure variation at isolator exit for all cases.

Total pressure distribution at the exit of the isolator for all the cases are presented in Fig. 15. Overall total pressure rise is noticed in both the cases when comparing with the base case.

\section{CONCLUSION}

Modifications of the cowl of an inlet and isolator geometry of a Mach 4 dual mode scramjet has been studied numerically. The simulation of the base case is validated with existing data from the literature. Introducing a concavity in the cowl is explored. The cowl wall is modified to have a circular arc concavity of two different depths, viz., 0.05 inch and 0.1 inch. The concavity is started at 0.5 inched from the cowl lip and ended at the throat of the intake. The changes in the shock pattern in the compression region and isolator are captured using numerical schlieren. The changes in the static pressure profile, total pressure recovery, Mach number and flow distortion are studied. The concavity causes expansion fans which weakens the cowl lip shock after some length, and decreases the boundary layer separation. Further, the weak compression fan in the concavity brings back the Mach number, while the separation is suppressed. This leads to a large gain in total pressure. The shock train now gets stretched in the downstream direction, giving rise to overall slightly higher Mach number and consequently lower static pressure and higher total pressure. Pressure peaks along the ramp and cowl surfaces are noticed to be changing in magnitude and location of impingement as well. Since the beginning of the concavity near cowl leading edge produces expansion fans, only cowl leading edge shock turns towards the flow. Hence, the mode of operation still remains slightly subcritical. The mass captured is not affected and remains the same since the cowl lip is not affected by the modification. Thus the present study gives a new geometry modification that can give higher total pressure recovery without affecting the mass captured. The concavity of depth 0.1 inch was found to have the best total pressure recovery. Higher depths were not attempted as they make the cowl thickness very small and unmanageable structurally. Further optimisation of the concavity is possible in terms of the starting location and the ending location of the cavity.

\section{REFERENCES}

Creighton, S. and R. Hillier (2007). Experimental and computational study of unsteady hypersonic cavity flows. The Aeronautical Journal 111(1125), 673-688.

Emami S., C. A. Trexler, A. H. Auslender and J. P. Weinder (1995). "Experimental investigation of inlet combustor isolators for a dual mode scramjet at Mach number of 4" NASA technical paper no . NASA-TP-3502

Filippi, A. A. and B. W. Skews (2017). Supersonic flow fields resulting from axisymmetric internal surface curvature. Journal of Fluid Mechanics 831, 271-288.

Gai, S. L., H. Kleine and A. J. Neely (2015). Supersonic Flow over a Shallow Open Rectangular Cavity. Journal of Aircraft 52(2), 609-616.

Guvernyuk, S. V., A. F. Zubkov and M. M. Simonenko (2016). Experimental Investigation 
P. Senthilkumar and T. M. Muruganandam / JAFM, Vol. 13, No. 5, pp. 1601-1610, 2020.

of the Supersonic Flow over an Axisymmetric Ring Cavity. Journal of Engineering Physics and Thermophysics 89(3), 678-687.

Heiser, W. H. and D. T. Pratt (1994). Hypersonic Air-breathing Propulsion, AIAA Educational Series.

Janarthanam, S. and V. Babu (2012). Numerical simulations of the flow through the inlet and isolator of a Mach 4 dual mode scramjet. The Aeronautical Journal 116(1182), 833-846.

Lad, K. A., R. R. Vinil Kumar and A. Vaidyanathan (2018). Experimental Study of Subcavity in Supersonic Cavity Flow. AIAA Journal 56(5), 1965-1977.

Lawson, S. J. and G. N. Barakos (2011). Review of numerical simulations for high-speed, turbulent cavity flows. Progress in Aerospace Sciences, 47(3), 186-216.

Liscinsky, D., B. True and J. Holdeman (1993). Experimental investigation of crossflow jet mixing in a rectangular duct. 29th Joint Propulsion Conference and Exhibit.

Mccormick, D. C. (1993). Shock/boundary-layer interaction control with vortex generators and passive cavity. AIAA Journal 31(1), 91-96

Mohri, K. and R. Hillier (2011). Computational and experimental study of supersonic flow over axisymmetric cavities. Shock Fans 21(3), 175191.

Morgenstern, A. and N. Chokani (1994). Hypersonic flow past open cavities. AIAA Journal 32(12), 2387-2393.

Needham, D. A. (1965). Laminar separation in hypersonic flow. Ph.D. dissertation, Imperial
College London, London.

Needham, D. and J. Stollery (1966). Boundary layer separation in hypersonic flow. 3rd and 4th Aerospace Sciences Meeting, American Institute of Aeronautics and Astronautics, Reston, VA.

Palharini, R. C., T. J. Scanlon and C. White (2018). Chemically reacting hypersonic flows over 3D cavities: Flow field structure characterisation. Computers \& Fluids 165, 173-187.

Panigrahi, C., A. Vaidyanathan and M. T. Nair (2019). Effects of subcavity in supersonic cavity flow. Physics of Fluids 31(3), 036101.

Raja sekar, K., S. Jegadheeswaran, R. Kannan and P. Manigandan (2019). Perforated Wall in Controlling the Separation Bubble Due to Shock Wave -Boundary Layer Interaction. International Journal of Turbo \& Jet-Engines

Surujhlal, D. and B. W. Skews (2018) Twodimensional supersonic flow over concave surfaces. Shock Fans, 1-7.

Waltrup, P. J., F. S. Billg and R. D. Stockbridge (1982). Engine sizing and integration requirements for hypersonic air breathing missile applications, $A G A R D-C P-207(8)$.

Wan, D. and R. Guo (2007). Experimental Investigation of a Fixed-geometry Twodimensional Mixed-compression Supersonic Inlet with Sweep-forward High- light and Bleed Slot in an Inverted "X"-type Layout. Chinese Journal of Aeronautics 20(4), 304 312.

Zhuang, N., F. S. Alvi, M. B. Alkislar and C. Shih (2006). Supersonic Cavity Flows and Their Control. AIAA Journal 44(9), 2118-2128. 